PROCEEDINGS OF THE

AMERICAN MATHEMATICAL SOCIETY

Volume 126, Number 9, September 1998, Pages 2797-2803

S 0002-9939(98)04369-X

\title{
SUFFICIENT CONDITIONS FOR ONE DOMAIN TO CONTAIN ANOTHER IN A SPACE OF CONSTANT CURVATURE
}

\author{
JIAZU ZHOU
}

(Communicated by Christopher B. Croke)

\begin{abstract}
As an application of the analogue of C-S. Chen's kinematic formula in the 3-dimensional space of constant curvature $\epsilon$, that is, Euclidean space $\mathbb{R}^{3}, 3$-sphere $S^{3}$, hyperbolic space $\mathbb{H}^{3}(\epsilon=0,+1,-1$, respectively), we obtain sufficient conditions for one domain to contain another domain in either an Euclidean space $\mathbb{R}^{3}$, or a 3 -sphere $S^{3}$ or a hyperbolic space $\mathbb{H}^{3}$.
\end{abstract}

\section{$\S 1$. INTRODUCTION}

For a long time one is faced with the problem of fitting a rugby into a box. Generalizing this problem we ask: given two domains in a space $\mathbb{E}_{\epsilon}^{n}$ of constant curvature when can one be moved by an isometry "inside" the other. The answer should only depend on geometric invariants of the domains, such as volumes, surface areas and curvature integrals of the boundaries of domains. In the case of $\mathbb{E}^{2}$ sufficient conditions were given by Hadwiger [8], [9]. By using integral geometry, Grinberg, Ren and Zhou [12] were able to give a fairly simple sufficient condition for domains in a plane $\mathbb{E}_{\epsilon}^{2}$ of constant curvature, and one that is derived simultaneously for all $\epsilon$. The key idea is to use the isoperimetric deficit which was first invoked by Ren [3] in the Euclidean plane. Higher dimensional analogues were found by Zhou [14], [15], [16], [17] and Zhang [5] in the Euclidean case. As one might expect the inequalities involve many invariants.

The purpose of this paper is to give sufficient conditions for one domain to contain another domain in a 3-dimensional space $\mathbb{E}_{\epsilon}^{3}$ of constant curvature. We use the analog of C-S. Chen's formula [4], [17], i.e., kinematic formula for curvature in the space $\mathbb{E}_{\epsilon}^{3}$ of constant curvature. In general the domains $D_{i}$ and $D_{j}$ are assumed to be $C^{2}$-smooth. We also place an upper bound on the Euler-Poincaré characteristics of the intersections $D_{i} \cap g D_{j}$. The latter requirement can be avoided if one just considers smooth convex bodies $D_{i}$ and $D_{j}$.

\section{Analogue of C-S. Chen's kinematic formula in $\mathbb{E}_{\epsilon}^{3}$}

Let $M_{i}, M_{j}$ be two submanifolds in a homogeneous space $G / H$ and $I$ an invariant of the intersection submanifold $M_{i} \cap g M_{j}$. Let $d g$ be suitably normalized kinematic

Received by the editors April 25, 1996 and, in revised form, February 18, 1997.

1991 Mathematics Subject Classification. Primary 52A22, 53C65; Secondary 51M16.

Key words and phrases. Kinematic formula, transfer principle, Weingarden transformation, Gaussian curvature, convex body, domain, mean curvature, total geodesic curvature.

(C)1998 American Mathematical Society 
density of $G$. Evaluating the integral

$$
\int_{\left\{g \in G: M_{i} \cap g M_{j} \neq \emptyset\right\}} I\left(M_{i} \cap g M_{j}\right) d g
$$

in terms of invariants of $M_{i}$ and $M_{j}$ we obtain the so-called kinematic formula [1], [2], [3], [6], [14], [17].

Let $\Sigma$ be a closed surface of class $C^{2}$ in a 3 -dimensional Euclidean space $\mathbb{R}^{3}$. Denote by $R, H$, respectively, the Gaussian curvature, the mean curvature of $\Sigma$. The total Gaussian curvature, the total square mean curvature are, respectively, defined by

$$
\tilde{R}=\int_{\Sigma} R d \sigma, \quad \tilde{H}^{2}=\int_{\Sigma} H^{2} d \sigma
$$

Let $M_{i}$ and $M_{j}$ be two surfaces of class $C^{2}$ in $\mathbb{R}^{3}$. Assume that $M_{i}$ is fixed, $g M_{j}$ is moving under the isometry $g$. Let $d g$ be the kinematic density for $\mathbb{R}^{3}$, so normalized that the measure of all positions about a point is $8 \pi^{2}$. Let $\kappa_{\Gamma_{g}}$ denote the curvature of the intersection curve $\Gamma_{g}=M_{i} \cap g M_{j}$. Denote by $F_{k}$ the surface area, and $\tilde{R}_{k}, \tilde{H}_{k}^{2}$ the total Gaussian curvature, the total square mean curvature of $M_{k}(k=i, j)$, respectively. Then we have the following C-S. Chen's kinematic formula [4], [17]

$$
\int_{G}\left(\int_{M_{i} \cap g M_{j}} \kappa_{\Gamma_{g}}^{2} d s\right) d g=2 \pi^{3}\left\{\left(3 \tilde{H}_{i}^{2}-\tilde{R}_{i}\right) F_{j}+\left(3 \tilde{H}_{j}^{2}-\tilde{R}_{j}\right) F_{i}\right\} .
$$

Let $\mathbb{E}_{\epsilon}^{3}$ be the 3-dimensional simply connected space of constant curvature $\epsilon$ and $I S_{\epsilon}^{3}$ the group of orientation preserving isometries of $\mathbb{E}_{\epsilon}^{3}$ with the normalized invariant density $d g$. Let $\Sigma \subset \mathbb{E}_{\epsilon}^{3}$ be a $C^{2}$-smooth surface and $W$ the Weingarten transformation. That is, if $\mathbf{n}$ is the unit normal to $\Sigma$ then $W$ is the linear map defined on the tangent bundle $T(\Sigma)$ by

$$
W X:=-\nabla_{X} \mathbf{n},
$$

where $\nabla$ is the Riemannian connection of $\mathbb{E}_{\epsilon}^{3}$ and $X \in T(\Sigma)$ a tangent field.

The principal curvature of $\Sigma$ is defined as the eigenvalue of $W$. The mean curvature $H_{\Sigma}$ and the extrinsic Gauss curvature $R_{\Sigma}^{e}$ are, respectively, defined by

$$
H_{\Sigma}=\frac{1}{2} \operatorname{Tr}(W)=\frac{\lambda_{1}+\lambda_{2}}{2}, \quad R_{\Sigma}^{e}=\operatorname{det}(W)=\lambda_{1} \lambda_{2},
$$

where $\lambda_{1}, \lambda_{2}$ are eigenvalues of $W$.

By the Gauss equation in $\mathbb{E}_{\epsilon}^{3}$ the Gauss curvature $R_{\Sigma}$ and the extrinsic Gauss curvature $R_{\Sigma}^{e}$ are related by (see [7], [13])

$$
R_{\Sigma}=R_{\Sigma}^{e}+\epsilon .
$$

The transfer principle [6] tells us that the form of kinematic formula in a homogeneous space $G / H$ does not depend on the full group of isometries $G$, but only on the invariant theory of the isotropy subgroup $H$. For example, this transfer principle allows us to obtain a new kinematic formula in the space $\mathbb{E}_{\epsilon}^{n}$ from a kinematic formula which has been proved in a Euclidean space $\mathbb{R}^{n}$ without changing invariants that are independent of the curvature $\epsilon$ of the space. Therefore by the 
transfer principle Chen's kinematic formula holds in the 3-dimensional space $\mathbb{E}_{\epsilon}^{3}$ in the following form:

$$
\int_{I S_{\epsilon}^{3}}\left(\int_{M_{i} \cap g M_{j}} \kappa_{\Gamma}^{2} d s\right) d g=2 \pi^{3}\left(\left(3 \tilde{H}_{j}^{2}-\tilde{R}_{j}^{e}\right) F_{i}+\left(3 \tilde{H}_{i}^{2}-\tilde{R}_{i}^{e}\right) F_{j}\right),
$$

where $\kappa_{\Gamma}$ is the geodesic curvature of curve $\Gamma_{g}=M_{i} \cap g M_{j}$.

By use of the Gauss equation, the formula (6) can be rewritten in the following form ${ }^{1}$ :

$$
\begin{aligned}
& \int_{I S_{\epsilon}^{3}}\left(\int_{M_{i} \cap g M_{j}} \kappa_{\Gamma}^{2} d s\right) d g \\
& \quad=2 \pi^{3}\left(\left(3 \tilde{H}_{j}^{2}-\tilde{R}_{j}\right) F_{i}+\left(3 \tilde{H}_{i}^{2}-\tilde{R}_{i}\right) F_{j}+2 \epsilon F_{i} F_{j}\right) .
\end{aligned}
$$

If two surfaces $M_{i}, M_{j}$ are closed then by use of the Gauss-Bonnet formula

$$
\tilde{R}_{\Sigma}=2 \pi \chi(\Sigma),
$$

where $\chi(\cdot)$ is the Euler-Poincaré characteristic, we obtain the following type kinematic formula.

Theorem 1. Let $M_{k}(k=i, j)$ be two closed $C^{2}$-smooth surfaces in $\mathbb{E}_{\epsilon}^{3}$. Denote by $F_{k}, H_{k}$ the area and the mean curvature of $M_{k}$, respectively. Then we have

$$
\begin{aligned}
& \int_{I S_{\epsilon}^{3}}\left(\int_{M_{i} \cap g M_{j}} \kappa_{\Gamma}^{2} d s\right) d g \\
& \quad=2 \pi^{3}\left\{3\left(F_{i} \tilde{H}_{j}^{2}+F_{j} \tilde{H}_{i}^{2}\right)-2 \pi\left(F_{i} \chi\left(M_{j}\right)+F_{j} \chi\left(M_{i}\right)\right)+2 \epsilon F_{i} F_{j}\right\} .
\end{aligned}
$$

\section{§3. HADWIGER'S THEOREM IN SPACE $\mathbb{E}_{\epsilon}^{3}$}

Let $D_{i}$ and $D_{j}$ be two connected domains in space $\mathbb{E}_{\epsilon}^{3}$, bounded by surfaces $\partial D_{i}$ and $\partial D_{j}$, which we assume to be of class $C^{2}$. In the case of the 3 -sphere $S^{3}, \partial D_{i}$ and $\partial D_{j}$ are assumed to be connected and simply connected. Moreover, we assume that $D_{i}$ and $D_{j}$ are such that, for all $g \in G$, the group of orientation preserving isometries of $\mathbb{E}_{\epsilon}^{3}$, the Euler-Poincaré characteristic $\chi\left(D_{i} \cap g D_{j}\right)$ of the intersection $D_{i} \cap g D_{j}$ has an upper bound $N_{0}$, a finite integer. Let $V_{k}(k=i, j), F_{k}, \tilde{H}_{k}$ and $\tilde{H}_{k}^{(2)}$ be the volume of $D_{k}$, the surface area of $D_{k}$, the total mean curvature and the total square mean curvature of $\partial D_{k}$, respectively. We have

Theorem 2. For the domains in a space $\mathbb{E}_{\epsilon}^{3}$ of nonnegative curvature $\epsilon$ (that is, space with constant curvature $\epsilon=0,+1)$, let $F_{\max }=\frac{1}{2} \max \left\{F_{i}, F_{j}\right\}$. In the case of $S^{3}$, we assume that $F_{k}<4 \pi(k=i, j)$. Then a sufficient condition for $D_{i}$ to contain $D_{j}$ or for $D_{j}$ to contain $D_{i}$ is

$$
\begin{aligned}
& 8 \pi\left(V_{i} \chi\left(D_{j}\right)+V_{j} \chi\left(D_{i}\right)\right)+2\left(F_{i} \tilde{H}_{j}+F_{j} \tilde{H}_{i}\right)-\frac{N_{0} \cdot 2 \pi^{2}}{2 \pi-\epsilon F_{\max }} \\
& \quad \times\left\{F_{i} F_{j}\left[3\left(\tilde{H}_{i}^{2} F_{j}+\tilde{H}_{j}^{2} F_{i}\right)-2 \pi\left(F_{i} \chi\left(\partial D_{j}\right)+F_{j} \chi\left(\partial D_{i}\right)\right)+2 \epsilon F_{i} F_{j}\right]\right\}^{\frac{1}{2}}>0 .
\end{aligned}
$$

Moreover, if $V_{j} \leq V_{i}$, then $D_{j}$ can be contained in $D_{i}$.

\footnotetext{
${ }^{1}$ This formula, i.e., the analogue of C-S. Chen's formula, is due to R. Howard.
} 
Proof. S. S. Chern's fundamental formula, Santaló's formula, respectively, read [1]

$$
\begin{gathered}
\int_{I S_{\epsilon}^{3}} \chi\left(D_{i} \cap g D_{j}\right) d g=8 \pi^{2}\left(V_{i} \chi\left(D_{j}\right)+V_{j} \chi\left(D_{i}\right)\right)+2 \pi\left(F_{i} \tilde{H}_{j}+F_{j} \tilde{H}_{i}\right), \\
\int_{I S_{\epsilon}^{3}\left(\partial D_{i} \cap g \partial D_{j}\right)} L_{\Gamma_{g}} d g=2 \pi^{3} F_{i} F_{j},
\end{gathered}
$$

where $L_{\Gamma_{g}}$ is the volume of the intersection curve $\Gamma_{g}=\partial D_{i} \cap g \partial D_{j}$, i.e., the arc length of $\Gamma_{g}$. The curve $\Gamma_{g}$ may be composed of several components (i.e., each of those is a simply closed curve).

For a disktype surface of area $F$ bounded by a simple $C^{2}$-smooth curve $\Gamma$, we have the following Fenchel-Teufel formula [11]:

$$
\int_{\Gamma}\left|\kappa_{\Gamma}\right| d s \geq 2 \pi-\epsilon F
$$

where $\kappa_{\Gamma}$ is the geodesic curvature of $\Gamma^{2}$

By Hölder's inequality we have

$$
\begin{aligned}
& 2 \pi- \epsilon F_{\max } \leq \int_{\Gamma_{g}}\left|\kappa_{\Gamma_{g}}\right| d s \leq\left(\int_{\Gamma_{g}} 1^{2} \cdot d s\right)^{\frac{1}{2}}\left(\int_{\Gamma_{g}} \kappa_{\Gamma_{g}}^{2} d s\right)^{\frac{1}{2}} \\
&=\left(L_{\Gamma_{g}}\right)^{\frac{1}{2}}\left(\int_{\Gamma_{g}} \kappa_{\Gamma_{g}}^{2} d s\right)^{\frac{1}{2}},
\end{aligned}
$$

i.e.,

$$
2 \pi-\epsilon F_{\max } \leq\left(L_{\Gamma_{g}}\right)^{\frac{1}{2}}\left(\int_{\Gamma_{g}} \kappa_{\Gamma_{g}}^{2} d s\right)^{\frac{1}{2}} .
$$

Integrating both sides of the above inequality with respect to kinematic density $d g$ and using Hölder's inequality again we get

$$
\begin{gathered}
\left(2 \pi-\epsilon F_{\max }\right) \int_{I S_{\epsilon}^{3}\left(\partial D_{i} \cap g \partial D_{j} \neq \emptyset\right)} d g \leq \int_{I S_{\epsilon}^{3}\left(\partial D_{i} \cap g \partial D_{j}\right)}\left(L_{\Gamma_{g}}\right)^{\frac{1}{2}}\left(\int_{\Gamma_{g}} \kappa_{\Gamma_{g}}^{2} d s\right)^{\frac{1}{2}} d g \\
\leq\left(\int_{I S_{\epsilon}^{3}\left(\partial D_{i} \cap g \partial D_{j}\right)} L_{\Gamma_{g}} d g\right)^{\frac{1}{2}}\left(\int_{I S_{\epsilon}^{3}\left(\partial D_{i} \cap g \partial D_{j}\right)}\left(\int_{\Gamma_{g}} \kappa_{\Gamma_{g}}^{2} d s\right) d g\right)^{\frac{1}{2}} .
\end{gathered}
$$

Therefore by (9), (12) and (16) we have

$$
\begin{aligned}
& \int_{I S_{\epsilon}^{3}\left(\partial D_{i} \cap g \partial D_{j} \neq \emptyset\right)} d g \leq \frac{2 \pi^{3}}{2 \pi-\epsilon F_{\max }}\left\{F _ { i } F _ { j } \left[3\left(F_{i} \tilde{H}_{j}^{2}+F_{j} \tilde{H}_{i}^{2}\right)\right.\right. \\
&\left.\left.-2 \pi\left(F_{i} \chi\left(\partial D_{j}\right)+F_{j} \chi\left(\partial D_{i}\right)\right)+2 \epsilon F_{i} F_{j}\right]\right\}^{\frac{1}{2}} .
\end{aligned}
$$

From

$$
\int_{I S_{\epsilon}^{3}\left(D_{i} \cap g D_{j}\right)} \chi\left(D_{i} \cap g D_{j}\right) d g \leq N_{0} \int_{I S_{\epsilon}^{3}\left(D_{i} \cap g D_{j} \neq \emptyset\right)} d g
$$

\footnotetext{
${ }^{2}$ In the case of Euclidean space $\mathbb{R}^{3}$, the inequality is Fenchel's inequality. Inequality is due to Teufel when $\Gamma$ is a curve in a 3 -sphere $S^{3}$.
} 
we obtain the kinematic measure

$$
\begin{aligned}
m\{g & \left.\in I S_{\epsilon}^{3}: g D_{j} \subseteq D_{i} \quad \text { or } \quad g D_{j} \supseteq D_{i}\right\} \\
& =m\left\{g \in I S_{\epsilon}^{3}: D_{i} \cap g D_{j} \neq \emptyset\right\}-m\left\{g \in I S_{\epsilon}^{3}: \partial D_{i} \cap g \partial D_{j} \neq \emptyset\right\} \\
& =\int_{I S_{\epsilon}^{3}\left(D_{i} \cap g D_{j} \neq \emptyset\right)} d g-\int_{I S_{\epsilon}^{3}\left(\partial D_{i} \cap g \partial D_{j} \neq \emptyset\right)} d g \\
& \geq \frac{1}{N_{0}}\left[8 \pi^{2}\left(V_{i} \chi\left(D_{j}\right)+V_{j} \chi\left(D_{i}\right)\right)+2 \pi\left(F_{i} \tilde{H}_{j}+F_{j} \tilde{H}_{i}\right)\right]-\frac{2 \pi^{3}}{2 \pi-\epsilon F_{\max }} \\
& \times\left\{F_{i} F_{j}\left[3\left(\tilde{H}_{i}^{2} F_{j}+\tilde{H}_{j}^{2} F_{i}\right)-2 \pi\left(F_{i} \chi\left(\partial D_{j}\right)+F_{j} \chi\left(\partial D_{i}\right)\right)+2 \epsilon F_{i} F_{j}\right]\right\}^{\frac{1}{2}} .
\end{aligned}
$$

If this lower bound is nonnegative then one domain can be moved to contain another. We complete the proof of Theorem 2.

Theorem 3. Let $D_{i}, D_{j}$ be two domains in a hyperbolic space $\mathbb{H}^{3}$. Then a suffcient condition for $D_{i}$ to contain $D_{j}$ or for $D_{j}$ to contain $D_{i}$ is

$$
\begin{aligned}
& 8 \pi\left(V_{i} \chi\left(D_{j}\right)+V_{j} \chi\left(D_{i}\right)\right)+2\left(F_{i} \tilde{H}_{j}+F_{j} \tilde{H}_{i}\right) \\
& \quad-\pi N_{0}\left\{F_{i} F_{j}\left[3\left(F_{i} \tilde{H}_{j}^{2}+F_{j} \tilde{H}_{i}^{2}\right)-2 \pi\left(F_{i} \chi\left(\partial D_{j}\right)+F_{j} \chi\left(\partial D_{i}\right)\right)-2 F_{i} F_{j}\right\}^{\frac{1}{2}}>0 .\right.
\end{aligned}
$$

Moreover, if $V_{j} \leq V_{i}$, then $D_{j}$ can be contained in $D_{i}$.

Proof. For a disktype surface of area $F$ bounded by a $C^{2}$-smooth curve $\Gamma$ in the 3dimensional hyperbolic space $\mathbb{H}^{3}$, we have the following Brickell-Hsiung inequality [18]:

$$
\int_{\Gamma}\left|\kappa_{\Gamma}\right| d s \geq 2 \pi+F
$$

By Hölder's inequality and the weaker type of inequality (21) we have

$$
\begin{aligned}
2 \pi \leq & \int_{\Gamma_{g}}\left|\kappa_{\Gamma_{g}}\right| d s \leq\left(\int_{\Gamma_{g}} 1^{2} \cdot d s\right)^{\frac{1}{2}}\left(\int_{\Gamma_{g}} \kappa_{\Gamma_{g}}^{2} d s\right)^{\frac{1}{2}} \\
& \leq\left(L_{\Gamma_{g}}\right)^{\frac{1}{2}}\left(\int_{\Gamma_{g}} \kappa_{\Gamma_{g}}^{2} d s\right)^{\frac{1}{2}},
\end{aligned}
$$

i.e.

$$
2 \pi \leq\left(L_{\Gamma_{g}}\right)^{\frac{1}{2}}\left(\int_{\Gamma_{g}} \kappa_{\Gamma_{g}}^{2} d s\right)^{\frac{1}{2}} .
$$

Integrating both sides of inequalitiy $(23)$ over $I S_{-1}^{3}\left(\partial D_{i} \cap g \partial D_{j} \neq \emptyset\right)$ and using the analog of C-S. Chen's formula and Hölder inequality again we have

$$
\begin{gathered}
\int_{I S_{-1}^{3}\left(\partial D_{i} \cap g \partial D_{j} \neq \emptyset\right)} d g \leq \pi^{2}\left\{F _ { i } F _ { j } \left[3\left(F_{i} \tilde{H}_{j}^{2}+F_{j} \tilde{H}_{i}^{2}\right)\right.\right. \\
\left.\left.-2 \pi\left(F_{i} \chi\left(\partial D_{j}\right)+F_{j} \chi\left(\partial D_{i}\right)\right)-2 F_{i} F_{j}\right]\right\}^{\frac{1}{2}} .
\end{gathered}
$$


By (18), (11) and (24) we have

$$
\begin{aligned}
m\{g & \left.\in I S_{-1}^{3}: g D_{j} \subseteq D_{i} \quad \text { or } \quad g D_{j} \supseteq D_{i}\right\} \\
& =m\left\{g \in I S_{-1}^{3}: D_{i} \cap g D_{j} \neq \emptyset\right\}-m\left\{g \in I S_{-1}^{3}: \partial D_{i} \cap g \partial D_{j} \neq \emptyset\right\} \\
& =\int_{I S_{-1}^{3}\left(D_{i} \cap g D_{j} \neq \emptyset\right)} d g-\int_{I S_{-1}^{3}\left(\partial D_{i} \cap g \partial D_{j} \neq \emptyset\right)} d g \\
& \geq \frac{1}{N_{0}}\left[8 \pi^{2}\left(V_{i} \chi\left(D_{j}\right)+V_{j} \chi\left(D_{i}\right)\right)+2 \pi\left(F_{i} \tilde{H}_{j}+F_{j} \tilde{H}_{i}\right)\right] \\
& -\pi^{2}\left\{F_{i} F_{j}\left[3\left(\tilde{H}_{i}^{2} F_{j}+\tilde{H}_{j}^{2} F_{i}\right)-2 \pi\left(F_{i} \chi\left(\partial D_{j}\right)+F_{j} \chi\left(\partial D_{i}\right)\right)-2 F_{i} F_{j}\right]\right\}^{\frac{1}{2}} .
\end{aligned}
$$

Inequality (25) immediately leads to the proof of Theorem 3.

As in euclidean space we say that a subset $S$ of a 3 -sphere $S^{3}$ or a 3 -hyperbolic space $\mathbb{H}^{3}$ is convex if $P, Q \in S$ implies that the geodesic segment joining $P$ to $Q$ also lies in $S$. A nonempty, compact, convex set of $\mathbb{E}_{\epsilon}^{3}$ is called a convex body. If $D_{i}$ and $D_{j}$ are convex bodies, we have $\chi\left(\partial D_{i}\right)=\chi\left(\partial D_{j}\right)=2$ and $\chi\left(D_{i}\right)=\chi\left(D_{j}\right)=$ $\chi\left(D_{i} \cap g D_{j}\right)=1$ for almost all $g \in I S_{\epsilon}^{3}$. Then from Theorem 2 and Theorem 3 we immediately obtain the following

Theorem 4. For convex bodies $D_{i}$ and $D_{j}$ in a space $\mathbb{E}_{\epsilon}^{3}$ of nonnegative constant curvature $\epsilon$ (i.e., $\epsilon=0,+1$ ), let $F_{\max }=\frac{1}{2} \min \left\{F_{i}, F_{j}\right\}$. In the case of $S^{3}$, we assume $F_{k}<4 \pi(k=i, j)$. Then a sufficient condition for $D_{i}$ to contain, or to be contained in, $D_{j}$ is

$$
\begin{aligned}
8 \pi\left(V_{i}\right. & \left.+V_{j}\right)+2\left(F_{i} \tilde{H}_{j}+F_{j} \tilde{H}_{i}\right)-\frac{2 \pi^{2}}{2 \pi-\epsilon F_{\max }} \\
& \times\left\{F_{i} F_{j}\left[3\left(\tilde{H}_{i}^{2} F_{j}+\tilde{H}_{j}^{2} F_{i}\right)-4 \pi\left(F_{i}+F_{j}\right)+2 \epsilon F_{i} F_{j}\right]\right\}^{\frac{1}{2}}>0 .
\end{aligned}
$$

Moreover, if $V_{i} \leq V_{j}$, then $D_{i}$ can be contained in $D_{j}$.

Theorem 5. Let $D_{i}$ and $D_{j}$ be convex bodies in a hyperbolic space $\mathbb{H}^{3}$. Then a sufficient condition for $D_{i}$ to contain, or to be contained in, $D_{j}$ is

$$
\begin{aligned}
8 \pi\left(V_{i}\right. & \left.+V_{j}\right)+2\left(F_{i} \tilde{H}_{j}+F_{j} \tilde{H}_{i}\right) \\
& -\pi\left\{F_{i} F_{j}\left[3\left(F_{i} \tilde{H}_{j}+F_{j} \tilde{H}_{i}\right)-4 \pi\left(F_{i}+F_{j}\right)-2 F_{i} F_{j}\right]\right\}^{\frac{1}{2}}>0 .
\end{aligned}
$$

Moreover, if $V_{j} \leq V_{i}$, then $D_{j}$ can be contained in $D_{i}$.

\section{ACKNOWLEDGEMENT}

We would like to thank Professor Eric Grinberg for many helpful discussions. We thank Professor Ralph Howard for pointing out to us that the generalized C-S. Chen's formula comes directly from his transfer principle. We would also like to thank our colleagues in Sultan Qaboos University for some comments. Finally, we

thank Professor C. C. Hsiung and the referee very much for many useful comments which made substantial improvements of the style of this paper. Also, Theorem 3 is an outcome of their comments.

\section{REFERENCES}

1. L. A. Santaló, Integral Geometry and Geometric Probability, Addison-Wesley, Reading, MA (1976). MR 55:6340

2. S. S. Chern and Chih-Ta Yen, Formula principale cinematica dello spazio ad $n$ dimensioni, Boll. Un. Mat. Ital. 2 (1940), 434-437. MR 3:89b 
3. Delin Ren, Topics in Integral Geometry, Singapore World Scientific International Publisher (1992). MR 96h:53087

4. C-S. Chen, On the kinematic formula of square of mean curvature, Indiana Univ. Math. J. 22 (1972-73), 1163-1169. MR 47:2529

5. G. Zhang, A sufficient condition for one convex body containing another, Chin. Ann. of Math. 9B(4) (1988), 447-451. MR 90k:52008

6. R. Howard, The kinematic formula in riemannian geometry, Memoir of the Amer. Math. Soc. 509 (1993).

7. Michael Spivak, A Comprehensive Introduction to Differential Geometry (II), Publish or Perish, Inc. (1979). MR 82g:53003b

8. H. Hadwiger, Genenseitige Bedeckbarkeit zweier Eibereiche und Isoperimetrie, Viertejsch. Naturforsch. Gesellsch. Zürich 86 (1941), 152-156. MR 4:112c

9. H. Hadwiger, Überdeckung ebener Bereiche derch Kreise und Quadrate, Comment. Math. Helv. 13 (1941), 195-200. MR 3:90f

10. Yu. D. Burago \& V. A. Zalgaller, Geometric Inequalities, Springer-Verlag Berlin Heidelberg (1988). MR 89b:52020

11. E. Teufel, On the total absolute curvature of closed curves in spheres, Manuscripta Mathematical 57 (1986), 101-108. MR 88b:53080

12. Eric Grinberg, Delin Ren \& Jiazu Zhou, The isoperimetric inequality and the containment problem in the plane of constant curvature, submitted.

13. B-Y Chen, Geometry of Submanifolds, Marcel Dekker Inc. New York (1973). MR 50:5697

14. Jiazu Zhou, Kinematic formulas for mean curvature powers of hypersurfaces and Hadwiger's theorem in $R^{2 n}$, Trans. Amer. Math. Soc. 345 no. 1 (1994), 243-262. MR 95a:52009

15. Jiazu Zhou, The sufficient condition for a convex body to contain another in $R^{4}$, Proc. Amer. Math. Soc. 121 no. 3 (1994), 907-913. MR 94i:52007

16. Jiazu Zhou, When can one domain enclose another in $R^{3}$ ?, J. Austral. Math. Soc. (Series A) 59 (1995), 266-272. MR 96f:52008

17. Jiazu Zhou, A kinematic formula and analogues of Hadwiger's theorem in space, Cont. Math. (Amer. Math. Soc.) 140 (1992), 159-167. MR 93k:52003

18. F. Brickell \& C. C. Hsiung, The total absolute curvature of closed curves in riemannian manifolds, J. Diff. Geom. 9 (1974), 177-193. MR 49:3795

19. Y. Tsukamoto, On the total absolute curvature of closed curves in manifolds of negative curvature, Math. Ann. 210 (1974), 313- 319. MR 51:1670

Department of Mathematics, Sultan Qaboos University, P.O.Box 36, Al-Khod 123, Sultanate OF OMAN

Current address: Department of Mathematics, Lehigh University, Bethlehem, Pennsylvania 18015-3174

E-mail address: jiz3@lehigh.edu 